\title{
Performance Analysis of a Multiuser Multipath Communication System: a Game Theoretical Approach
}

\section{Béla Almási*, Tamás Balogh**, János Kormos*, Balázs Kreith*}

* Faculty of Informatics, University of Debrecen, Kassai u. 26, H-4028 Debrecen, Hungary, almasi.bela@inf.unideb.hu,kormos.janos@inf.unideb.hu, kreith.balazs@inf.unideb.hu

** Faculty of Economics and Business, University of Debrecen, Böszörményi u. 138, H-4032 Debrecen, Hungary, tamas.balogh@econ.unideb.hu

\begin{abstract}
The study of multipath communication technologies is a hot research area today. One natural effect of using multipath communication instead of the single path one is the higher throughput value which will result in a better performance, not only in the usual Internet communication, but also in Big Data centers where the communication infrastructure can appear as a bottleneck point of the system. In this paper, we introduce a new game theoretical model for the evaluation of multiuser-multipath communication technologies. The decision problem for the users (i.e. network clients) is studied in a multipath communication system. We develop a game theoretical model for client payoff maximization, where the decision variables for each client are defined as their path requests. Due to limited hardware performance and limited service capacity, we assume that each client's payoff depends on other clients' path requests. We apply the tools of game theory to describe equilibrium behavior of the clients in the given interaction situation. By providing two examples, we show that our model is suitable for measuring payoffs, both in money and in throughput. We also offer possible directions for the further development of our model.
\end{abstract}

Keywords: multipath communication; throughput aggregation; Data Center Networks; game theory modeling; concave games; performance analysis 


\section{Introduction}

\subsection{Overview of the Multipath Communication Technologies}

The traditional Internet Communication Technology (IP), uses a single communication path between the endpoints in a communication session, since the IP address of the endnode is a part of the socket ID. In the case of multipath routing (see e.g. [1]), when the packet transmission in the Cloud is distributed by routers among multiple paths (in order to decrease the effect of congestion), the endnode uses only one of its interfaces, in the communication session. Thus, a communication session is connected to a single interface of the endnode. The currently used devices (laptops, tablets and mobile phones) usually have more than one network interface: Wi-Fi, 3 G/4 G, Bluetooth, NFC etc. (see Figure 1 below for illustration).

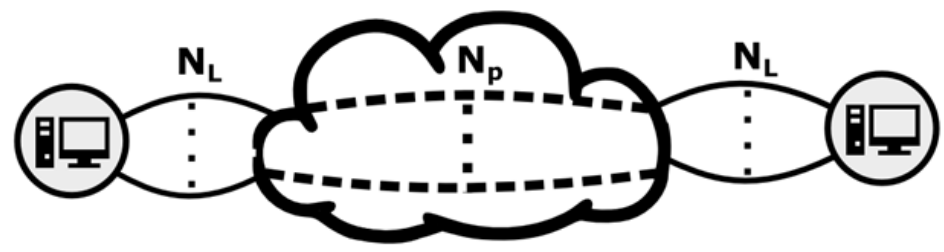

Figure 1

Illustration of multipath-multilink communication networks

The idea of combining available interfaces in one communication session is an important research topic today. The advantage of aggregating the throughput capacity of multiple interfaces is a widely used technology, also in Big Data centers where the communication infrastructure may become the bottleneck point of the overall system's performance [2]. In the modern Data Center Networks (DCNs) the throughput sensitive large flows can be effectively serviced by using multipath infrastructure background, which can be tuned according to the DCN special requirements (see [3]).

The multipath communication technology is studied in different OSI Layers: The IEEE "Convergent Digital Home Network" working group started the creation of the 1905.1 standard at the Data Link Layer. It is a special implementation of the Multipath technology (it is named as "Multilink technology" in Layer 2, as there is no multihop path in this case [4]). The 1905.1 standard focuses on the throughput aggregation possibility of the different links. The IETF RFC 6824 document "TCP Extensions for Multipath Operation with Multiple Addresses" [5] was published in January 2013. It specifies the extensions of the traditional TCP Transport Layer protocol (named as MPTCP) in order to be able to use multiple paths in one communication session. The aim of the MPTCP specification is to 
increase the throughput and the reliability of the TCP communication session. The MPT software library roughly targets as the MPTCP, but works at the Network Layer. One common purpose of the multipath/multilink technology is to aggregate the speed capacity of the different paths/links (i.e. throughput aggregation). Different laboratory measurements show (see [6], [7], [8]) that the multipath technology is able to efficiently aggregate the throughput capacity of the paths. In [6] Paasch et al. introduced an MPTCP based system, which produced a World Record throughput capacity of $50 \mathrm{Gbps}$ by aggregating 6 pieces of $10 \mathrm{Gbps}$ connection paths. Almási and Szilágyi showed an MPT based system in [7] and [8] aggregating 2 and 4 pieces of paths with the efficiency ratio better than $95 \%$ in each case.

Lencse and Kovács investigated the aggregation performance limits of the MPT multipath software library in [9] and [10]: The throughput capacity of the multipath system increased linearly with the number of paths using IPv4 (up to the systems' maximum of 12 paths with speed of 100 Mbps in each physical connection). Using IPv6, the test measurements reached the maximum limit of the aggregation in the same laboratory environment: The throughput of the system increased up to 7 paths, but then no further speed increasing could be seen even when adding more paths to the system. Considering the performance evaluation, this limitation was caused by the hardware. The overall throughput performance remained roughly constant after a special number of allocated paths. It means that allocating more paths to the system did not increase the overall throughput performance.

The performance evaluation of the multipath communication systems, their usability, utilization and consequences is also an actual research area. The utilization of MPTCP according to the client throughput aggregation to the overall network communication system is investigated in Khalili, Ramin, et al. [11]. They analyzed the performance of the MPTCP [5] communication system and in contrast to TCP based communications, they proved that it was not Paretooptimal. A system is called Pareto-optimal if the improvement of an individual participant's benefit is impossible without decreasing another one's. In contrast to MPTCP, MPT uses a different path scheduling algorithm for link aggregation, where Pareto-optimality is not proved, but currently it serves as a basis of further investigation.

Due to the interaction of endnodes and the server in a MPT network, for modeling the overall system utilization, a game theoretical approach is also adequate.

Game theory is fundamental for modeling and evaluating systems in which the overall benefits (payoffs) of the participants depend on their individual decisions. Seminal contributions can be found in $[12,13]$. Studying network communication systems by using a game theoretical approach, clients are assumed to choose their path request strategies in order to reach the best overall performance. Applying game theory tools for different network problems takes place frequently, see e.g. 
[14], where cooperative and non-cooperative approaches are compared for network problems. Increasing the network capacity indefinitely and letting the routers decide the best path individually may lead to Braess's Paradox [15]. Braess's Paradox describes that in certain circumstances in which participants are choosing the best path individually without cooperation, they can actually slow down the network. It concludes that the existence of multiple paths between two nodes in the network in some circumstances can produce worse overall system utilization than using fewer paths.

The overall system utilization investigation using game theory suggests strategies on how to design or use the overall network system. As Lencse and Kovács [9] show, hardware capacity influences the aggregated bandwidth limitation at the client site, it leads us to investigate the overall system utilization further and to use game theory approaches.

A precise mathematical model of the multipath network communication system using game theoretical tools may open the possibility for many investigations related to the overall system utilization. It can be used for instance to investigate and model the path allocation strategies for multipath communication systems according to the client requests and the server interests. Other investigations may also be performed focusing on utilization and payoff interests of the participants of the system.

In this paper we introduce a non-cooperative game-theoretical framework for path allocation in a multipath network communication system to provide an analytical tool for finding the best available path allocation mechanism in the system. We combine a classic game theoretical approach with the multipath network communication system, in which clients aim at increasing their communication speeds by requesting new paths from the server. After defining the model and proving existence of equilibrium, we present two example solutions. In the first, clients are assumed to maximize their monetary payoffs which is defined as a linear combination of their utility of receiving a certain number of paths (expressed in money), and the financial costs for their path requests. The second approach considers the limited hardware capacity of the client and aims at maximizing throughput. Thus, in the second example, the payoff is not expressed as money, but as throughput.

We emphasize that the two examples employ two different, but adequate approaches to model client behavior and therefore, we do not wish to show any preferences. They can be considered as suggestions to understand and solve clients' decision problem in a multipath communication environment.

We note that our model does not yet take server payoff maximization into consideration, in order to provide clear results on the client site. A possible further research direction includes investigating the server side's decision problem. However, the server is involved in our model as well, but its role is restricted to allocate paths according to a certain allocation rule. 
The main contribution of this paper is to offer a link between game theory and multipath communication systems and introduce a model that is suitable for further studies.

The rest of the paper is organized as follows... Section 2 provides the mathematical specification of multipath communication networks. Section 3 defines the path allocation game for a multipath system, offering a link between multipath communication networks and game theoretical models, where game theory is used to model the decision problem and strategic interaction of the clients in a multipath environment. Section 4 introduces two theoretical game approaches and that match the concept introduced in Section 3. We also provide the equilibrium scenario of the two games. Finally, Section 5 concludes our work.

\section{Mathematical Specification of Multiuser-Multipath Systems}

We define the following notations for representing the parameters of the multipath communication systems.

$\mathrm{N}_{\mathrm{L}}$ : The number of physical links that connect the Node (client) to the Internet.

$\mathrm{K}$ : The maximum number of paths used by the multipath communication system

$\mathrm{L}_{\mathrm{i}}$ : The speed (bandwidth) of the $\mathrm{i}^{\mathrm{th}}$ physical link, which connects the Node to the Internet

$\mathrm{U}_{\mathrm{P}}$ : The throughput capacity of one path (homogeneous system)

MPSpeed(k): The speed (throughput capacity) of the system aggregating k paths.

$\operatorname{Aggr}(\mathrm{k})$ : The aggregation efficiency of aggregating k paths:

$$
\operatorname{Aggr}(k)=\frac{\operatorname{MPSPeed}(k)}{k \cdot U_{P}}
$$

The number of physical links $\left(\mathrm{N}_{\mathrm{L}}\right)$ is limited by the interface number of the Node. As the multipath communication system may include not totally disjoint paths (i.e. paths with common links), the number of paths may be larger than the number of physical links (i.e. $N_{L}<N_{P}$ ) even in the case of efficient aggregation (see [7], [8]).

Of course, the sum of the physical links' speeds gives a limit for the theoretical maximum speed of the multipath system: $N_{P} \cdot U_{P} \leq \sum_{i=1}^{N_{L}} L_{i}$. 
As it was presented in [9], the aggregation needs resources from the Node (mainly CPU). The available resources are limited on the Node:

ResMax ${ }_{\mathrm{i}}$ : The maximum of the available resources on the $\mathrm{i}^{\text {th }}$ Node.

For simplicity, we may assume that the resource need of aggregating $k$ paths $($ denoted by $\operatorname{Res}(\mathrm{k}))$ is linearly increasing by $\mathrm{k}: \operatorname{Res}(k)=k \cdot \operatorname{Res}$.

For all k the inequality $\operatorname{Res}(k) \leq \operatorname{ResMax}$ must hold for each client $i$.

PMax $_{\mathrm{i}}$ : The maximal available path the Node can handle according to ResMax $\mathrm{x}_{\mathrm{i}}$. If resources are infinite, then the maximal number of path the system can handle is limited by the bandwidth of the physical link: $\operatorname{PMax}_{i}=\frac{\sum_{j=1}^{N_{L}} L_{j}}{U_{p}}$.

In what follows, we define a theoretical game framework to analyze Node (client) behavior. Later on, in Section 4 we present two models that are in coherence with the mathematical specification of the multipath communication framework.

\section{The Game Theoretical Framework}

We define a non-cooperative game theoretical approach for the allocation of paths in the multi-user environment. We give a formal model for Node (i.e. client, household) payoff maximization in a multipath communication environment. The main idea is that each client aims at maximizing its own profit (which we will call payoff in accordance with the game theoretic terminology). The payoff function of a certain node - precisely defined below - consists of a function of the node's total throughput and a cost function of the requested paths.

In our simplified framework, at the beginning of the allocation process, each endnode announces simultaneously the requested amount of paths. Then, according to an allocation rule, paths are assigned to the endnodes and the payoff functions are evaluated.

\subsection{Definition and Assumptions of the Game}

The strategic (normal) form of the game is as follows.

\section{Definition 3.1 (Path allocation game)}

The players are the endnodes which we denote by $1,2 \ldots \mathrm{n}$. The strategy set of any player i is given by $S(i)=\{0,1,2 \ldots \mathrm{K}\}$, where a strategy stands for the (integer) number of requested paths, which can grow up to $\mathrm{K}$. K stands for the maximum 
number of available paths for the whole system. For any $i$, we denote the requested number of paths by $\mathrm{k}_{\mathrm{i}}$. The payoff function of any player $i$ is given by:

$$
\pi_{i}\left(k_{1}, k_{2} \ldots k_{n}\right)=f_{i}\left(k_{1}, k_{2} \ldots k_{n}\right)-c_{i}\left(k_{i}\right)
$$

where $f_{i}$ is the utility of player $i$ (expressed in money or throughput). Note that $f_{i}$ depends directly on the received number of paths, and indirectly - as indicated in the arguments of $f_{i}$ - on the requests of all endnodes. Throughout this section, for the sake of simplicity we refer to $f_{i}$ as depending directly on the requested amounts of paths. At the same time, $c_{i}$ is a cost function of the requested paths of player $i$.

We note that throughout the analysis we relax the logical assumption of $\mathrm{k}_{\mathrm{i}}$ being an integer in order to create continuous payoff functions. Provided that the solution is not an integer number for a certain player (endnode), we will assume that the endnodes will choose the integer value resulting in the closest payoff level to optimal.

Before giving the solution of the game, we introduce the following assumptions on the payoff functions.

The first assumption is in accordance with the principle of decreasing marginal utility. This principle can also be applied for consumers (endnodes) in the communication environment, as a certain growth in bandwidth is worth more if the initial bandwidth of a user is smaller.

Assumption 3.2. For any $i, f_{i}$ is strictly increasing, continuous in $k_{i}$ on $[0 \ldots \mathrm{K}]$, strictly concave and twice continuously differentiable on its domain.

The following assumption dictates that if an endnode increases its number of paths requested, then its extra cost is more if the initial request was larger. This ensures that the amount of paths a household needs costs proportionally less than industrial consumption.

Assumption 3.3. For any $i$, the financial cost function $c_{i}$ is strictly increasing in $k_{i}$, convex and twice continuously differentiable on its domain.

From Assumptions 3.2 and 3.3 it is trivial that the combined payoff function of each player is concave in its own path request variable. We state this in the following corollary.

Corollary 3.4. For any $i$, the combined $\pi_{i}$ is concave and twice continuously differentiable in $k_{i}$. 
If the total number of requested paths is too large, then the server cannot satisfy all needs. We define a rationing (allocation) rule to determine the number of received paths as a function of all path requests for each endnode.

Definition 3.5. Rationing (allocation) rule:

For any $i$,

$$
R_{i}\left(k_{1}, k_{2} \ldots k_{n}\right)=\left\{\begin{array}{l}
k_{i} \quad \text { if } \sum_{i=1}^{n} k_{i} \leq K \\
\frac{K k_{i}}{\sum_{i=1}^{n} k_{i}} \text { if } \sum_{i=1}^{n} k_{i}>K
\end{array}\right.
$$

This means that whenever the sum of requests exceeds the available paths, paths are allocated in proportion of the requests.

We note that according to Definition 3.1 endnodes pay according to the number of their requested paths (and not the received ones).

We also note that whenever the sum of path requests does not exceed $K$, the payoff functions depend only on one variable (which is the client's own path request).

Finally, we assume that every player is aware of the strategies and payoff functions of all the others. This is in accordance with the assumption that "neighbors" know each other well enough to be able to estimate their needs.

Assumption 3.6. For any $i$, the set of players, $S(i)$ and $\pi_{i}$ are common knowledge.

Our last assumption considers the issue of time. For simplicity reasons we assume that the game is played as a one-shot game, excluding time elapse from the model. A suitable extension of this model to real-life situations is to consider the repeated version of the game.

Now we fix the ordering of actions in the one-shot game.

Step 1. Every endnode announces its requested amount of paths simultaneously by sending the need to the server.

Step 2. After receiving the needs, the server allocates the paths to the endnodes according to the rationing rule.

It follows directly that the endnodes are not aware of each other's requests at the moment they have to send their own need to the server. 


\subsection{Equilibrium of the Path Allocation Game}

To find the payoff-maximizing strategy of each endnode, we will employ the Nash equilibrium concept.

According to the definition, the Nash equilibrium players choose mutually beneficial response strategies for each other. (For more about the Nash equilibrium concept, we refer the reader to [16].) For the multipath environment, this means that in equilibrium every endnode is satisfied with its received amount of paths, because none of them would be able to increase their payoff level $\left(\pi_{i}\right)$ by ceteris paribus modifying its own request.

In what follows, we cite a useful theorem for the concave games and show that the path allocation game has exactly, one Nash equilibrium in pure strategies. After proving existence, we determine the unique Nash equilibrium profile.

Theorem 3.7 (Rosen). Equilibrium of concave games (from Theorems 1 and 2 of [17])

Let us consider a game of $\mathrm{n}$ players given in normal form. If for any $i$ the payoff function $\pi_{i}$ is strictly concave in player $i$ 's own decision variable, then the game has one and only one Nash equilibrium strategy profile in pure strategies.

The proof can be found in [17].

Corollary 3.8. The path allocation game has one and only one Nash equilibrium in pure strategies.

The proof follows directly from the assumptions that for any endnode $i, f_{i}$ is strictly concave, while $c_{i}$ is convex in $k_{i}$.

To determine the single equilibrium of the path allocation game, we use the fact that Nash equilibrium strategies are best responses for each other, thus, for any $i$, $\pi_{i}$ is maximized by the choice of the equilibrium strategy, whenever the strategies of the other players are fixed at their equilibrium levels.

Formally, this means that the one and only one Nash equilibrium profile of the game is implicitly provided as a solution of an equation system of $n$ equations.

Proposition 3.9. The following system of equations provides implicitly the only Nash equilibrium profile of the path allocation game.

$$
\frac{\partial \pi_{1}}{\partial k_{1}}=0 ; \quad \frac{\partial \pi_{2}}{\partial k_{2}}=0 \quad \ldots \quad \frac{\partial \pi_{n}}{\partial k_{n}}=0
$$

From the previous result, a question might arise. Namely, for which of the function classes does the above system have an explicit solution? We omit the 
mathematical analysis of this problem, as in the following section, we will provide numerical results for different types of payoff functions.

In the following Section 4, we present two approaches to handle client payoff. The first one considers monetary payoffs, while in the second one, payoffs are given in throughput. Both games will match the framework we presented in Section 3, and also the features of the systems defined earlier in Section 2.

\section{Models of Client Behavior}

As defined in Section 3, a participant's total payoff depends on its (positive) utility and the cost function value. In a multipath communication system a client may use multiple interfaces and logical paths in order to maximize its throughput efficiency. In the case of several multipath communication (MPC) clients being connected to a common multipath capable server that has finite resources (e.g.: limited number of physical interfaces and bandwidth) the server must distribute the resources among the clients by considering their requests. According to this given allocation rule, every client wants to maximize their individual payoff regardless of the others. As described in Section 3, based on the requests sent by the clients to the server, the server distributes the requested resources. The requests can be throughput needs or a number of logical paths that the client wants to use, etc. The sum of the requests may exceed the available resources.

In what follows, a game is defined for two clients as players $(1,2)$. They send their throughput requests $\left(\mathrm{R}_{1}, \mathrm{R}_{2}\right)$, to the MPT server, which calculates the number of paths to allocate to them based on their requests. For simplicity we assume, as previously described in Section 2, that a number of used resources linearly increases by the number of allocated paths distributed to the players. A player, by sending its throughput request and then participating in the game, must consider that other clients send their requests to the server as well. Every client has their own cost function limiting their payoffs. Whenever the server allocates paths from a finite resource that is exceeded by the sum of client needs, in equilibrium, one player's payoff may increase only by decreasing another player's payoff.

We note here, that the models presented, can easily be generalized for the morethan-two player cases, using the concept described in Section 3.

The payoff functions are defined as follows:

$$
\begin{aligned}
& \pi_{1}\left(k_{1}, k_{2}\right)=f_{1}\left(\mathrm{R}_{1}\left(k_{1}, k_{2}\right)\right)-c_{1}\left(k_{1}\right) \\
& \pi_{2}\left(k_{1}, k_{2}\right)=f_{2}\left(\mathrm{R}_{2}\left(k_{1}, k_{2}\right)\right)-c_{2}\left(k_{2}\right)
\end{aligned}
$$


Here, $k_{i}$ denotes the value of request of the $i$ th player, $\mathrm{R}_{\mathrm{i}}$ indicates the allocation rule used by the server for distributing the paths according to the players' requests, function $f_{i}$ transfers throughput to utility (e.g. expressed in money) and $c_{i}\left(k_{i}\right)$ denotes $i^{\text {th }}$ player's cost function.

If the above payoff functions are used in the framework game defined in Section 3 , one and only one Nash equilibrium point exists in the game. In that case the Nash equilibrium point can be determined by finding the only mutual best response strategies of the two players, as described in Section 3. Technically, this means that both players maximize their payoffs taking into consideration the other player's strategy. Thus, the following system of equations has to be solved:

$$
\begin{aligned}
& \frac{\partial \pi_{1}\left(k_{1}, k_{2}\right)}{\partial k_{1}}=0 \\
& \frac{\partial \pi_{2}\left(k_{1}, k_{2}\right)}{\partial k_{2}}=0
\end{aligned}
$$

To calculate the equilibrium after every request has arrived to the server, we need to find the numerical solution of the equation system defined above.

In what follows, we present two examples with different payoff functions and different focus. In the first one, MPC clients aim at maximizing their individual payoffs expressed in money. This game serves as an example of how an MPC client aims at increasing its payoff related to the throughput it receives, while the payoff is limited by the cost function. In the second game, payoff functions are represented by throughput gains and the cost functions are represented as hardware resource limitations caused by the increasing number of requested paths as investigated by Lencse and Kovacs [9].

\subsection{Monetary Payoffs}

In this subsection we present the analytical solution of a simple, two-player version of the path allocation game. We assume that the capacity of the server is 8 paths (i.e. $\mathrm{K}=8$ ). The payoff functions of the two endnodes are as follows ( $\pi_{i}$ always consists of a utility function and a financial cost function).

$$
\pi_{1}\left(k_{1}, k_{2}\right)= \begin{cases}10 K \frac{k_{1}}{k_{1}+k_{2}}-k_{1}^{2} & \text { if } k_{1}+k_{2}>K \\ 10 k_{1}-k_{1}^{2} & \text { if } k_{1}+k_{2} \leq K\end{cases}
$$




$$
\pi_{2}\left(k_{1}, k_{2}\right)= \begin{cases}10 K \frac{k_{1}}{k_{1}+k_{2}}-k_{2}^{3 / 2} & \text { if } k_{1}+k_{2}>K \\ 10 k_{2}-k_{2}^{3 / 2} & \text { if } k_{1}+k_{2} \leq K\end{cases}
$$

It is easy to see that the first (utility) part in the payoff function is strictly concave for both players, while the cost part is strictly convex. Therefore, $\pi_{i}$ remains strictly concave for both players.

To decide which payoff functions provide the equilibrium, we have to solve the equation system generated by the simpler one and check whether the sum of path requests are below 8 or not. From the latter payoff functions we obtain

$$
\begin{aligned}
& \frac{\partial \pi_{1}\left(k_{1}, k_{2}\right)}{\partial k_{1}}=10-2 k_{1}=0 \\
& \frac{\partial \pi_{2}\left(k_{1}, k_{2}\right)}{\partial k_{2}}=10-\frac{3}{2} \sqrt{k_{2}}=0
\end{aligned}
$$

It follows directly that in equilibrium, $k_{1}=5 ; k_{2}=44 \frac{4}{9}$. The sum of the requests exceeds 8 , therefore the former, more complicated payoff functions, should be used to find the equilibrium. Thus, we obtain the following system of equations:

$$
\begin{aligned}
& \frac{\partial \pi_{1}\left(k_{1}, k_{2}\right)}{\partial k_{1}}=80 \frac{k_{2}}{\left(k_{1}+k_{2}\right)^{2}}-2 k_{1}=0 \\
& \frac{\partial \pi_{2}\left(k_{1}, k_{2}\right)}{\partial k_{2}}=80 \frac{k_{1}}{\left(k_{1}+k_{2}\right)^{2}}-\frac{3}{2} \sqrt{k_{2}}=0
\end{aligned}
$$

We obtain, that in equilibrium, $k_{1}=3.041 ; k_{2}=5.334$. If we require integer values, then the solution is 3 and 5 , respectively. This result is seemingly far from that of the former equation system, but we note that the previous solution did not take into consideration the server's capacity limit. And finally, it does not matter, where the unconditional optimal level lies, if the sum of requests exceeds $K$. The following table presents the payoff function values of the two players when receiving certain amounts of paths.

Clearly, the only Nash equilibrium point is played if Player 1 requests 3 paths, while Player 2 requests 5 paths. This can easily be justified by analyzing the previous Table 1 . Here, at the $(3 ; 5)$ allocation profile, it is in neither of the players interest to alter their strategies individually, while the other player's strategy remains fixed. This is because neither of the players can increase their payoff by a unilateral modification of her request. 
Table 1

Payoff function values according to the number of requested paths of players $1 ; 2$

\begin{tabular}{|l|c|c|c|c|c|}
\hline \multicolumn{1}{|c|}{$\mathrm{k} 2$} & 2 & 3 & 4 & 5 & 6 \\
\hline $\mathrm{k} 1$ & & & & & \\
\hline 2 & $16.00 ; 17.17$ & $16.00 ; 24.80$ & $16.00 ; 32.00$ & $16.00 ; 38.82$ & $16.00 ; 45.30$ \\
\hline 3 & $21.00 ; 17,17$ & $21.00 ; 24.80$ & $21.00 ; 32.00$ & $\mathbf{2 1 . 0 0 ; 3 8 . 8 2}$ & $17.67 ; 38.64$ \\
\hline 4 & $24.00 ; 17.17$ & $24.00 ; 24.80$ & $24.00 ; 32.00$ & $19.56 ; 33.26$ & $16.00 ; 33.30$ \\
\hline 5 & $25.00 ; 17.17$ & $25.00 ; 24.80$ & $19.47 ; 27.56$ & $15.00 ; 28.82$ & $11.36 ; 28.94$ \\
\hline 6 & $24.00 ; 17.17$ & $17.33 ; 21.47$ & $12.00 ; 24.00$ & $7.64 ; 25.18$ & $4.00 ; 25.30$ \\
\hline
\end{tabular}

The following figures illustrate the payoffs of player 1 and 2 based on the previous results.

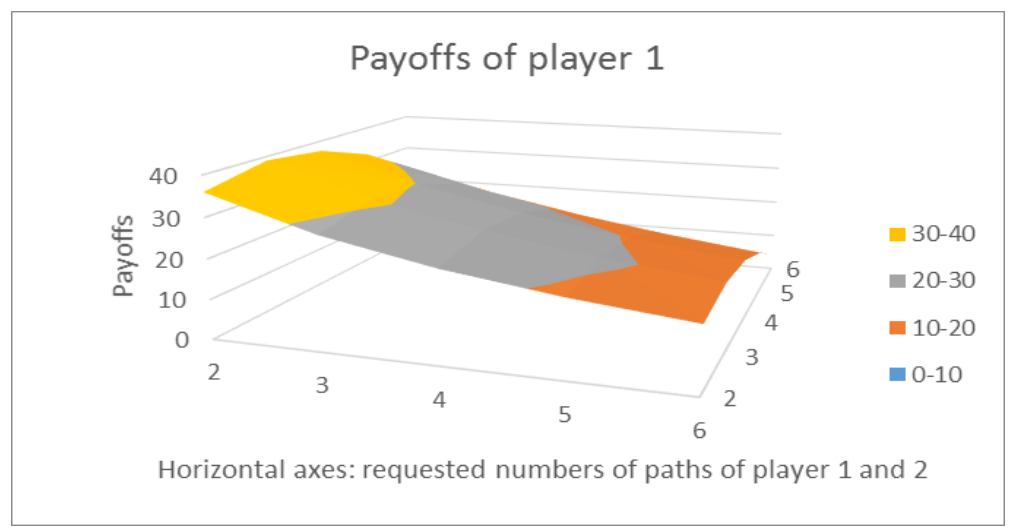

Figure 2

Monetary payoffs of player 1

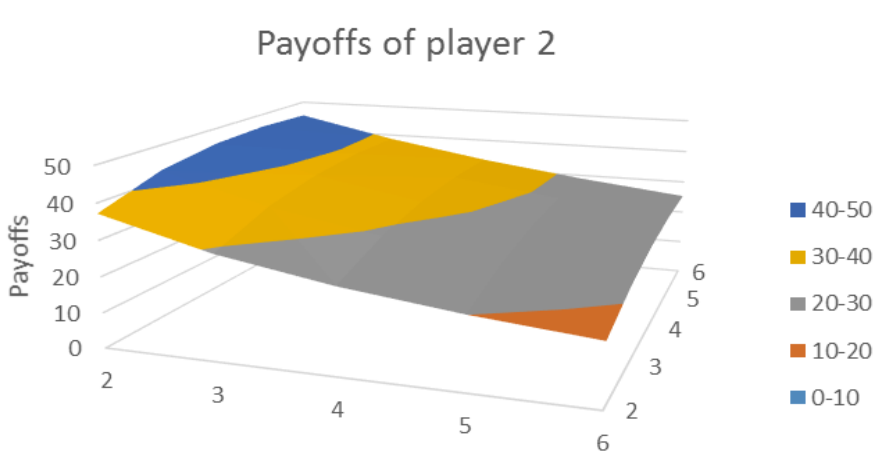

Horizontal axes: requested numbers of paths of player 1 and 2

Figure 3

Monetary payoffs of player 2 
It is visible from the figures that the discussed game belongs to the family of concave games. This is because, if we fix the number of requested paths for a player, then the payoff function of the other player is concave in its own variable. The only Nash equilibrium lies at the profile of requested paths where both players obtain maximum possible payoffs provided that the other player's choice is fixed. The importance of Theorem 3.7, lies in the unique nature of the Nash equilibrium profile.

As it can also be seen, the server can calculate the Nash equilibrium point based on the throughput requests the players sent to it, and thus, the server is also involved in the process, as declared in the introduction.

In the following example, we will change the payoff functions and use throughput increases and decreases representing the utility and cost values.

\subsection{Throughput Payoffs}

In the previous example the server calculated the number of paths to be given to the players based on the throughput needs and monetary payoff calculation. Based on the assumptions we argued that multipath communication system path allocation strategy for finite resources can be modeled by game theory and the path distribution is calculated according to the players' benefits. In the following we create a game between players sharing common limited resources and requests for paths in order to maximize their own throughput capacity, but their overall increases are limited by the hardware capacity they have. The utility is represented by the gained throughput and the cost function is represented by the loss of the throughput, for an MPC client. We use the observation described by Lencse and Kovacs in [9] that the throughput aggregation of a client that uses multipath communication system is limited by the hardware capacity. In this game, the cost function representing this limitation appeared after a certain number of paths. The payoff function increases quasi-linearly according in the received path number, and remains quasi-constant after it reaches its hardware limitation.

A suitable function that represents what we defined in the previous paragraph is defined below as the cost function of the client.

$$
\begin{aligned}
& \pi_{1}\left(k_{1}, k_{2}\right)=0.01\left(p\left(k_{1}\right) U_{p}-\ln \left(1+e^{p\left(k_{1}\right)-P M a x_{1}}\right)\right) \\
& \pi_{2}\left(k_{1}, k_{2}\right)=0.01\left(p\left(k_{2}\right) U_{p}-\ln \left(1+e^{p\left(k_{2}\right)-P M a x_{2}}\right)\right)
\end{aligned}
$$

Here, $k_{1}, k_{2}$ represent the number of requested paths of players 1 and 2 , respectively. $U_{p}$ is the total throughput capacity of the server, which is used by the players. The 0.01 multiplier is used only to avoid large function values. The $p\left(k_{i}\right)$ in the utility part of the function (14) and (15) denotes the possessed number of paths the $i$ th player acquired from the server. If the sum of the number 
of requested paths $\left(k_{1}+k_{2}\right)$ does not exceed the total number of available path $(K)$, then $p\left(k_{i}\right)=k_{i}$. If the sum of the requested paths exceeds those available, $K$, the server must distribute the paths in proportion to the requests originated from the players (we refer the reader to the rationing (allocation) rule defined in Section 3). In that case, $p\left(k_{i}\right)=\left\lfloor k_{i} K /\left(k_{1}+k_{2}\right)\right\rfloor$. The cost function is the throughput loss for the $i$ th player that happens above a certain number of allocated paths, caused by the hardware limitation of the client. The maximal number of paths the player can handle is determined by $\operatorname{PMax}_{i}$. The cost function $\ln \left(1+e^{p\left(k_{i}\right)-P M a x_{i}}\right)$ is a softplus sigmoid function. This function increases quasilinearly until a certain point $\left(\operatorname{PMax}_{i}\right)$ and then remains quasi-constant. Its usability and the property of being easily differentiable makes this function applicable to represent the hardware limitation that occurs at a certain point, where the MPC client acquires a number of paths that exceeds its limitation.

It is easy to see that the game is concave, as the utility part of each payoff function is concave, while the cost part is convex. Therefore, we can apply Theorem 3.7 and state that this game has again one and only one Nash equilibrium point.

To determine the one and only Nash equilibrium profile of the game, we have to solve the following system of equations.

$$
\begin{aligned}
& \frac{\partial \pi_{1}\left(k_{1}, k_{2}\right)}{\partial k_{1}}=\left(\left\lfloor\frac{2 k_{1}+k_{2}}{K^{2}}\right\rfloor U_{p}-\frac{1}{1+e^{p\left(k_{1}\right)-P M a x_{1}} p\left(k_{1}\right) d k_{1}}\right) 0.01 \\
& \frac{\partial \pi_{2}\left(k_{1}, k_{2}\right)}{\partial k_{2}}=\left(\left\lfloor\frac{2 k_{1}+k_{2}}{K^{2}}\right\rfloor U_{p}-\frac{1}{1+e^{p\left(k_{2}\right)-P M a x_{2}} p\left(k_{2}\right) d k_{2}}\right) 0.01
\end{aligned}
$$

The payoff function of players remains quasi-constant after they acquired the number of paths given by PMax $_{i}$. That payoff function is applicable for modeling the throughput increases, according to the players path requests, from the server in a multipath communication system. The following illustrates the hardware resource changes, by changing the requested number of paths.

We fixed $P M a x_{1}$ and $P M a x_{2}$ to both players for 6 and 5 respectively. The throughputs of the players regarding their requests are presented in the following tables and figures. The Nash equilibrium profile is also indicated. 
Table 2

Payoff function values according to the number of requested paths of players $1 ; 2$

\begin{tabular}{|l|c|c|c|c|c|}
\hline \multicolumn{1}{|c|}{ k2 } & 2 & 3 & 4 & 5 & 6 \\
\hline 21 & & & & & \\
\hline 3 & $19.51 ; 19.51$ & $19.51 ; 28.73$ & $19.51 ; 36.86$ & $19.51 ; 43.06$ & $19.51 ; 46.86$ \\
\hline 4 & $29.51 ; 19.51$ & $29.51 ; 28.73$ & $29.51 ; 36.86$ & $29.51 ; 43.06$ & $19.81 ; 43.06$ \\
\hline 5 & $48.73 ; 19.51$ & $38.73 ; 28.73$ & $38.73 ; 36.86$ & $29.51 ; 36.86$ & $29.51 ; 36.86$ \\
\hline 6 & $53.06 ; 19.51$ & $46.86 ; 28.73$ & $46.86 ; 28.73$ & $38.73 ; 28.73$ & $\mathbf{3 8 . 7 3 ; 3 6 . 8 6}$ \\
\hline
\end{tabular}

The following figures illustrate the payoffs of player 1 and 2 based on the previous results.

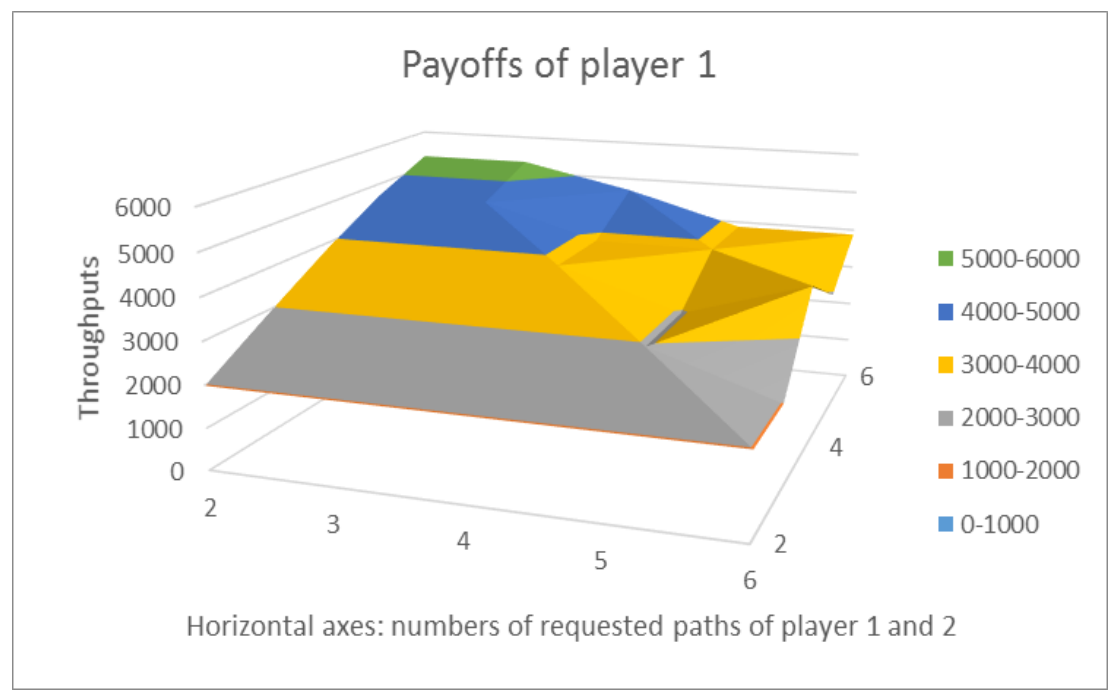

Figure 4

Throughput payoffs of player 1 


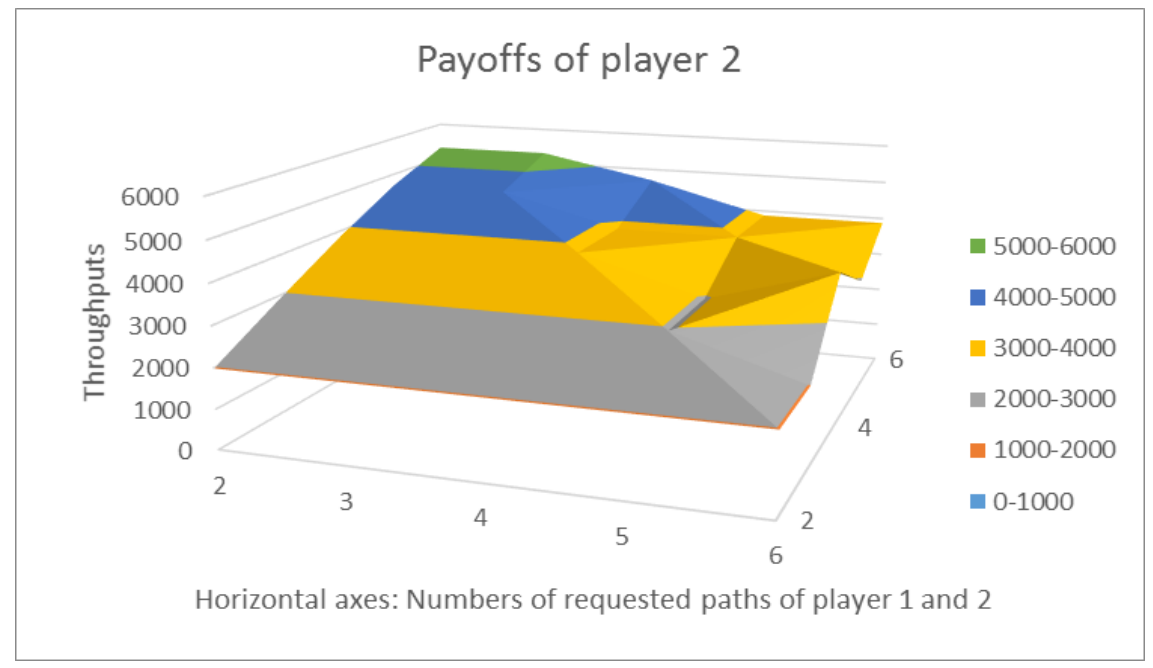

Figure 5

Throughput payoffs of player 2

We can draw the same conclusion as in Section 4.1. As the game is concave, we have only one Nash equilibrium profile of path requests. The payoff functions of both players are strictly concave, if the request of the other player is fixed.

The models can easily be extended to more than two players. As presented in Section 3, the construction of the equation system, leading to the unique Nash equilibrium profile for the game remains the same, for more than two endnodes.

\section{Conclusions}

These current systems usually have more than one networking interface: Wi-Fi, $3 \mathrm{G} / 4 \mathrm{G}$, Bluetooth, NFC etc. The idea of combining available interfaces in one communication session is a hot research area today. Multipath/multilink technology aims at aggregating speed capacities - i.e. throughputs - of the available paths/links. Different laboratory measurements show that the multipath technology, is able, to efficiently aggregate the throughput capacity of the paths.

Due to the interaction of endnodes and the server in a multipath/multilink environment, we found that a game theoretical approach could also be useful for modeling the overall system utilization. In this paper we introduced a noncooperative, game-theoretical framework, for path allocation in a multipath network communication system to provide an analytical tool for finding the best available path allocation mechanism in the system. We applied a classical game theoretical approach for the multipath/multilink network communication system, in which clients aim at increasing their bandwidths by requesting new paths from the server. 
After a precise mathematical definition of the multipath/multilink environment, we introduced our game-theoretical model. We stressed the use of the Nash equilibrium concept throughout the paper. We showed that the defined game belonged to the class of concave games, ensuring the existence of a single Nash equilibrium strategy profile in the game. Our results were proven to applicable for a wider range of payoff functions. Therefore, we had the possibility to present two different examples to solve a path allocation game. In the first, clients were assumed to maximize their monetary payoffs, which was defined as a linear combination of their utility of receiving a certain number of paths (expressed in money), and their financial cost for their path requests. The second approach considered the limited hardware capacity of the clients. In the second example, the payoff was not expressed in money, but in throughput which can be used also to investigate modeling of Big Data centers' performance, using multipath internal communication infrastructure (see [2]).

For both games a rationing (allocation) rule was defined. We illustrated the payoff vectors of the two endnodes for different path request combinations, and besides, we gave explanation for the single (unique) Nash equilibrium. The two example models employed two different approaches, to model client behavior, and we did not wish to show a preference to either side. These can be considered as suggestions, to understand and solve clients' decision problem, in a multipath/multilink environment. Our models can be considered as clear, positive results for client side payoff maximization. A possible further research direction includes investigating the server side's decision problems. Another direction might include a more exact specification (estimation) of clients' payoff functions, based on lab experiments.

To conclude, this paper has contributed to multipath/multilink network research, by offering a link between game theory and multipath/multilink systems, and has introduced a model that is suitable for further studies.

\section{Acknowledgement}

The work was supported by the TÁMOP-4.2.2.C-11/1/KONV-2012-0001 project. The project has been supported by the European Union, co-financed by the European Social Fund.

\section{References}

[1] M. L. M. Kiah, L. K. Qabajeh, M. M. Qabajeh, "Unicast Position-based Routing Protocols for Ad-Hoc Networks", Acta Polytechnica Hungarica, Vol. 7. No. 5, pp. 19-46, 2010

[2] C. Raiciu, S. Barre, C. Pluntke, A. Greenhalgh, D. Wischik, M. Handley, "Improving Datacenter Performance and Robustness with Multipath TCP", Proceedings of the ACM SIGCOMM 2011 conference, pp. 266-277, ACM New York, NY, USA, 2011 
[3] Y. Cao, M. Xu, X. Fu, E. Dong, "Explicit Multipath Congestion Control for Data Center Networks", Proceedings of the CoNEXT'13, pp. 73-84, 2013, Santa Barbara, California, USA, 2013

[4] IEEE Standards Association, "1905.1-2013 - IEEE Standard for a Convergent Digital Home Network for Heterogeneous Technologies", 2013. Available: http://standards.ieee.org/findstds/standard/1905.1a2014.html (Downloaded: 12/01/2015)

[5] A. Ford, C. Raiciu, M. Handley, O. Bonaventure, "TCP Extensions for Multipath Operation with Multiple Addresses"; IETF RFC-6824, 2013. Available: http://tools.ietf.org/html/rfc6824 (Downloaded 20/01/2015)

[6] C. Paasch, G. Detal, S. Barré, F. Duchêne, O. Bonaventure: "The Fastest TCP Connection with Multipath TCP"; ICTEAM, UCLouvain, Louvain-laNeuve, Belgium; http://multipath-tcp.org/pmwiki.php?n=Main.50Gbps (Downloaded: 04/03/2015.)

[7] B. Almási, Sz. Szilágyi: "Throughput Performance Analysis of the Multipath Communication Library MPT", Proceedings of the $36^{\text {th }}$ International Conference on Telecommunications and Signal Processing (TSP 2013, ISBN:978-1-4799-0403-7), pp. 86-90, Rome, Italy, 2013

[8] B. Almási, Sz. Szilágyi: "Investigating the Throughput Performance of the MPT Multipath Communication Library in IPv4 and IPv6", Journal of Applied Research and Technology, To appear

[9] G. Lencse, Á. Kovács, "Advanced Measurements of the Aggregation Capability of the MPT Multipath Communication Library", International Journal of Advances in Telecommunications, Electrotechnics, Signals and Systems, Vol. 4. No. 2, pp. 41-48, 2015

[10] G. Lencse, Á. Kovács, "Testing the Channel Aggregation Capability of the MPT Multipath Communication Library", World Symposium on Computer Networks and Information Security 2014 (WSCNIS 2014), Hammamet, Tunisia, 13-15 June, 2014, ISBN: 978-9938-9511-9-6, Paper ID: 1569946547

[11] Khalili, Ramin, et al. "MPTCP is not Pareto-Optimal: Performance Issues and a Possible Solution." Proceedings of the $8^{\text {th }}$ international conference on Emerging networking experiments and technologies. ACM, 2012

[12] Morgenstern, Oskar, and John Von Neumann. "Theory of Games and Economic Behavior" (1953)

[13] Nash Jr, John F. "The Bargaining Problem." Econometrica: Journal of the Econometric Society (1950): 155-162

[14] Easley, David, and J. Kleinberg. "Modeling Network Traffic using Game Theory."Networks, Crowds, and Markets: Reasoning about a Highly Connected World (2010): 229-247 
[15] Murchland, John D. "Braess's Paradox of Traffic Flow." Transportation Research4.4 (1970): 391-394

[16] Fudenberg, Tirole, J. "Game Theory" MIT Press, 1991

[17] J. B. Rosen. "Existence and Uniqueness of Equilibrium Points for Concave N-Person Games" Econometrica, Volume 33, Issue 3, 1965, pp. 520-534 